\title{
UNA VISIÓN TOULMINIANA RESPECTO A LA DISCIPLINA INVESTIGACIÓN BÁSICA EN EDUCACIÓN EN CIENCIAS: EL ROL DEL FORO INSTITUCIONAL
}

\author{
A Toulminian vision regarding to the discipline of basic \\ research in science education: the role of the institutional forum
}

\author{
Marco Antonio Moreira ${ }^{2}$
}

\begin{abstract}
Resumen: El objectivo de esta presentación es el argumentar que el área de investigación en educación en ciencias ha alcanzado un nivel de desarrollo que ya puede ser considerada una disciplina según los criterios de Stephen Toulmin. Por lo tanto, el foro institucional constituído por los grupos de referencia, las asociaciones de investigación, los periódicos y los cursos de postgrado del área deben asumir su importante papel en la continuidad de ese desarrollo. Al final son presentadas las conclusiones y recomendaciones de dos congresos de investigación en educación en ciencias que plantean cuestiones relevantes para dicho foro.
\end{abstract}

Unitermos: educación en ciencias, investigación básica, foro institucional.

\begin{abstract}
The purpose of this paper is to argue that the area of research in science education has reached a level of development that it might be already considered a discipline according to the criteria proposed by Stephen Toulmin. Thus, the institutional forum made up of reference groups, research associations, research journals, and graduate courses in this area must assume its important role in the continuation of such a development. Finally, the conclusions and recommendations of two meetings on research in science education are presented as relevant issues that should be handled by this forum.
\end{abstract}

Keywords: science education, basic research, institutional forum.

\section{Introducción}

Este texto corresponde a una misma ponencia hecha para investigadores en educación en ciencias en dos encuentros del área. La razón de publicarla es para que quede como documento de trabajo, es decir, de discusión, en el ámbito del foro institucional del area. Por un lado, el área de investigación en educación en ciencias es propuesta como una disciplina y, por otro, son identificadas debilidades, planteados desafíos y hechas recomendaciones para el desarrollo de dicha disciplina. Aunque dichas debilidades, desafíos y recomendaciones resulten de solamente dos congresos del área, creo que son muy útiles como temas para discusión. Analogamente, las visiones personales, y la interpretación toulminiana respecto a la investigación básica en educación en ciencias, presentadas en este trabajo, pretenden tan sólo contribuir para la discusión crítica de la investigación en el área. Todos eses puntos son aquí propuestos como temas en debate.

Inicialmente intentaré aclarar qué es lo que entiendo por investigación básica en educación en ciencias a través de una definición y tres diagramas esquemáticos. Después presentaré algunos criterios toulminianos para caracterizar una disciplina, su consolidación y su evolución. Al final, las conclusiones de dos encuentros iberoamericanos sobre investigación básica en educación en ciencias serán propuestos como tema de discusión en grupos de investigación, encuentros de investigadores, asociaciones y cursos de postgrado en educación en ciencias.

\footnotetext{
${ }^{1}$ Ponencia hecha en el $7{ }^{\circ}$ Simposio de Investigación en Educación en Física, 7 a 9 de octubre de 2004, Santa Rosa, La Pampa, Argentina y en la apertura de la II Semana de Investigación del Programa Internacional de Doctorado en Enseñanza de las Ciencias UBU/UFRGS, 18 a 22 de octubre de 2004, Porto Alegre, Brasil.

${ }^{2}$ Instituto de Física/UFRGS. Caixa Postal: 15051 - Cep: $91501-970$ - Porto Alegre, RS, Brasil. www.if.ufrgs.brl - moreira
} 


\section{¿Qué es investigación básica en educación en ciencias?}

Es la producción de conocimientos sobre educación en ciencias: búsqueda de respuestas a preguntas sobre enseñanza, aprendizaje, currículum y contexto educativo en ciencias, así como sobre el profesorado de ciencias y su formación permanente, dentro de un cuadro epistemológico, teórico y metodológico consistente y coherente en el cual el contenido específico de las ciencias está siempre presente. Será este el significado que usaré de aquí en adelante al referirme a la investigación en educación en ciencias.

\section{Tres diagramas esquemáticos.}

Las figuras 1, 2 y 3 presentan tres diagramas que intentan dar una visión esquemática de la investigación en educación en ciencias. El primero de ellos, en la figura 1, es la conocida $\mathrm{V}$ Epistemológica, o diagrama V, un dispositivo heurístico creado por D. B. Gowin (1981) para representar el proceso de producción de conocimiento, destacando en ese proceso, la interacción entre el pensar (dominio conceptual y epistemológico) y el hacer (dominio metodológico). En esta figura, la $\mathrm{V}$ epistemológica está aplicada a la investigación en educación en ciencias, desde una perspectiva bastante amplia.

La figura 2 es un mapa conceptual para la investigación en educación en ciencias. Un mapa conceptual es un diagrama jerárquico de conceptos y relaciones entre conceptos. Precisamente eso es lo que se intenta hacer en esta figura, con relación a los conceptos incluidos en la investigación en educación en ciencias. En ese tipo de diagrama priorizase siempre la estructura conceptual de un cuerpo de conocimientos, o de un dominio cognitivo, no su completud.

$\mathrm{La}$ tercera de estas figuras es nuevamente una $\mathrm{V}$ epistemológica, o un diagrama $\mathrm{V}$, pero incluso más detallado que el de la figura 1. Obsérvese que en este diagrama están incluidos ejemplos de filosofías, epistemologías, teorías, principios y conceptos usados en el dominio conceptual y epistemológico, sin intención alguna de darles mayor valor a unos que a otros. En el dominio metodológico, se incluyen los dos principales enfoques a la investigación educativa: el cualitativo y el cuantitativo. En el centro de la $\mathrm{V}$ se relacionan más o menos en orden cronológico las principales preguntas que se han planteado los investigadores en enseñanza de las ciencias desde la década de los años setenta, cuando la investigación en esa área toma un gran impulso. No se trata, no obstante, de una lista exhaustiva.

A continuación serán presentadas excerptas de la obra La comprensión humana de Stephen Toulmin (1977) que tratan de criterios disciplinarios.

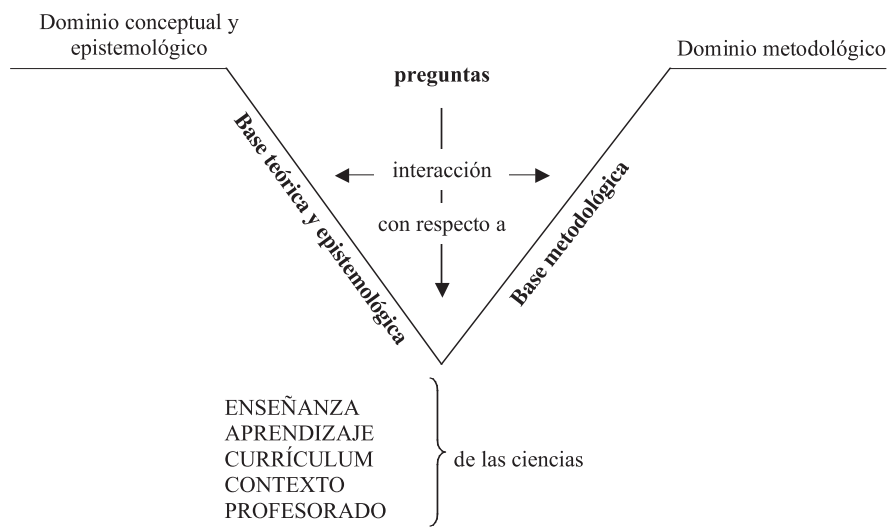

Figura 1 - Un diagrama $V$ de la investigación en educación en ciencias: una visión general. (MOREIRA, M. A., 2004) 


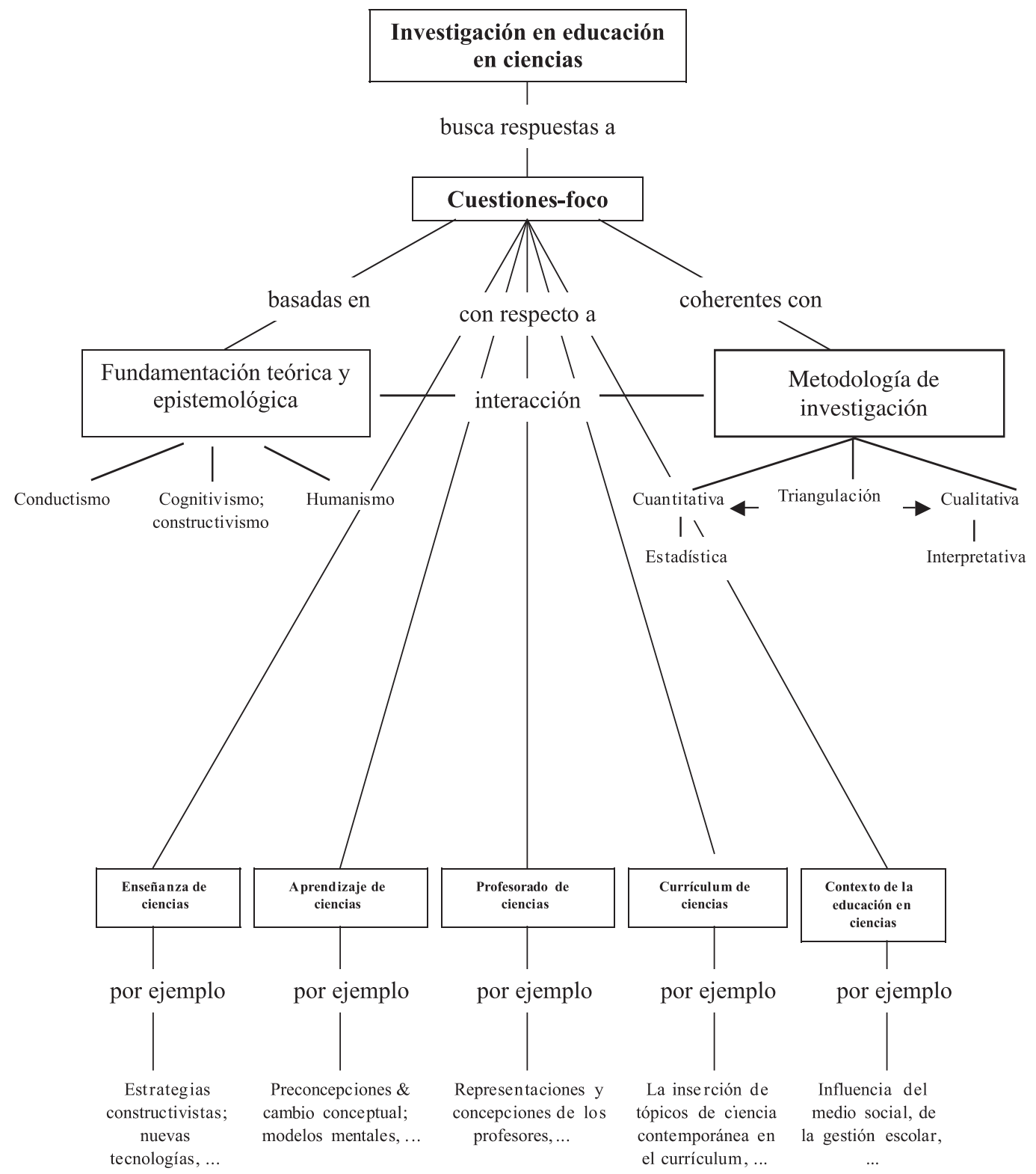

Figura 2 - Un mapa conceptual para la investigación en educación en ciencias. (MOREIRA, M. A., 1998, 2004) 
DOMINIO

CONCEPTUAL Y EPISTEMOLOGICO

\section{Filosofías}

(visiones del mundo, creencias profundas): p.ej., el conocimiento científico como construcción individual y colectiva; se puede mejorar la educación en ciencias através de la investigación en ese campo.

\section{Epistemologías y teorías:} p.ej., Popper, Kuhn, Lakatos, Laudan, Toulmin, ..., p.ej., Piaget, Ausubel, Vygostky,...

Representaciones Mentales,...

\section{Principios y con ceptos:}

p.ej., el alumno como constructor de su conocimiento; el cambio conceptual com o cambio de paradigmas; el cambio conceptual como evolución conceptual; la resolución de problemas como investigación; ...; esquema de asimilación, aprendizaje significativo, cambio conceptual, constructivismo,

represent ación mental, modelo mental, didáctica de las ciencias, formación de profesores, ...

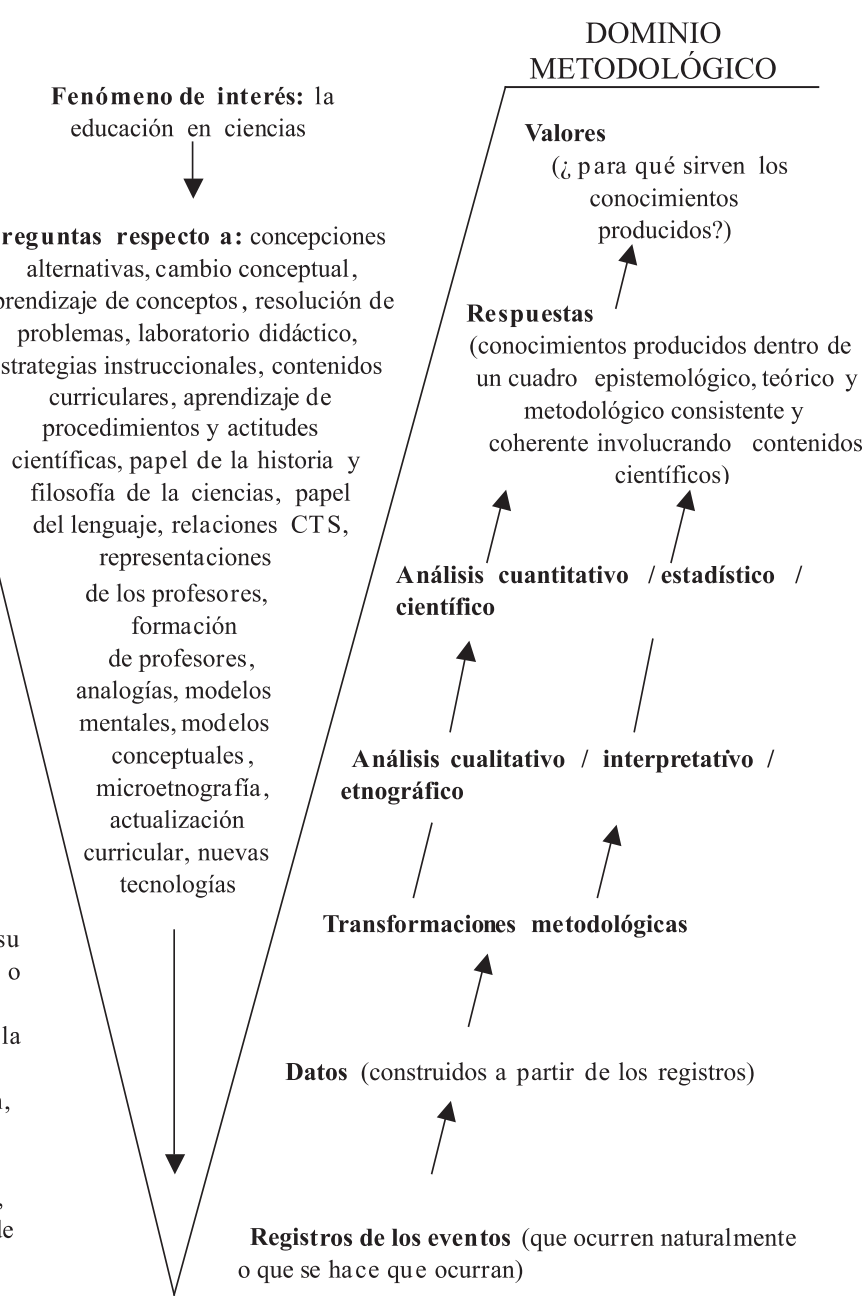

Objeto de estudio: la enseñanza, el aprendizaje y el currículum de ciencias; el contexto en que se da la educación en ciencias; el profesorado de ciencias y su formación permanente.

Figura 3 - Un diagrama $\mathrm{V}$ para la investigación en educación en ciencias: una visión detallada. (MOREIRA, M. A., 1998, 2004) 


\section{Disciplinas}

- Dentro de una cultura y época particular, las actividades intelectuales de los hombres no forman una gama continua desordenada. Por el contrario, caen en "disciplinas" más o menos bien definidas, caracterizada cada una de ellas por su propio cuerpo de conceptos, métodos y objetivos fundamentales... aunque mutable, cada disciplina normalmente exhibe una continuidad reconocible... por consiguiente, una explicación evolutiva del desarrollo conceptual tiene que explicar dos caracteres separados: por un lado, la coherencia y continuidad por la que identificamos las disciplinas como distintas y, por el otro, los profundos cambios a largo plazo por los que se transforman o son superadas. (op. cit., p. 149)

- En toda disciplina viva siempre hay novedades intelectuales que entran para su discusión al conjunto corriente de ideas y técnicas, pero sólo unas pocas de esas novedades conquistan un lugar firme en la disciplina y son transmitidas a las generaciones siguientes. La continua emergencia de innovaciones intelectuales se equilibra así como un continuo proceso de selección critica. Algunas variantes conceptuales son elegidas para su incorporación y otras son descartadas o ignoradas; pero, en circunstancias adecuadas, este mismo proceso puede explicar la continua estabilidad de una disciplina bien definida o su rápida transformación en algo nuevo y diferente... deben existir adecuados "foros de competencia" dentro de los cuales las novedades intelectuales puedan sobrevivir durante un tiempo suficiente para mostrar sus méritos o defectos, pero en el cual también son criticadas y escudriñadas con suficiente severidad como para mantener la coherencia de la disciplina. (p. 150)

- Las disciplinas cientificas, como las especies orgánicas, son "entidades históricas" en evolución y no "seres eternos" (ibid.)

* El elemento fundamental de una disciplina colectiva es el reconocimiento de un objetivo o ideal sobre el que existe suficiente acuerdo y en términos del cual es posible identificar los problemas comunes principales. Cuando este objetivo común es de carácter explicativo, la disciplina es científica. (p. 369)

- Contempladas como "empresas racionales" en desarrollo histórico dedicadas a la mejora de nuestros procedimientos educativos, y no como secuencias de sistemas preposicionales lógicamente estructurados, las disciplinas científicas están obligadas a su propia transformación. (p. 174)

- En un campo científico que aún no ha logrado el rango de disciplina, el debate teórico puede, a lo sumo, concentrarse en las grietas metodológicas reconocidas del campo... mientras una disciplina posible permanezca en esa condición incipiente y preliminar, no puede establecerse con autoridad ningún conjunto acordado de conceptos fundamentales o constelación de presupuestos básicos, ningún paradigma... (p. 385)

- Desde el momento en que un conjunto de conceptos logra una autoridad indiscutible en cualquier campo de la investigación, esa disciplina ya no se enfrenta con problemas "científicos" propiamente dichos y cesa de ser un campo de indagación cientifica. (p. 197) - Cualquier que sea el punto en el que estudiemos el proceso de la variación conceptual hallamos factores intrínsecos (o intelectuales) y extrínsecos (o sociales) que influyen sobre él conjuntamente, como dos filtros que actúan independientemente. Los factores sociales limitan las ocasiones y los incentivos para la innovación intelectual.

- Las debilidades institucionales de las disciplinas posibles pueden manifestarse de una variedad de formas. Los indicios más típicos son las deficiencias en la comunicación y la mala distribución de la autoridad. (p. 392)... una ciencia inmadura puede seguir siendo una "disciplina difusa" o "posible" por deficiencias institucionales (ibid). 
- La creación de grupos de referencia y periódicos autorizados desempeña un papel particularmente en la maduración de una disciplina posible. (p. 394)

- El contenido de una ciencia se transmite de una generación de cientificos a la siguiente por un proceso de enculturación. Este proceso supone un aprendizaje, por el cual ciertas habilidades explicativas se transfieren, con o sin modificación, de la generación más vieja a la más joven. En este aprendizaje, el núcleo de la transmisión - el elemento primario que debe ser aprendido, probado, aplicado, criticado, cambiado - es el repertorio de técnicas, procedimientos y habilidades intelectuales y métodos de representación que se emplean para "dar explicaciones" de sucesos y fenómenos dentro del ámbito de la ciencia involucrada. (p. 168, 169)

- Los individuos y las organizaciones ejercen, de hecho, un poder y una influencia tan reales sobre el desarrollo de la ciencia como en cualquier otra esfera de la vida humana. En correspondencia con eso, vale la pena luchar por los roles, cargos y posiciones de influencia de una profesión cientifica - y en la realidad se lucha por ellos - tan decidida, metódica y hasta tortuosamente como en cualquier otra esfera. (p. 273)

- Todos los miembros acreditados de una profesión cientifica pueden ser iguales en teoría; pero algunas resultan ser "más iguales" que otros. (p. 270)

\section{Periódicos}

- La misma razón de existir de muchas sociedades científicas reside principalmente en los periódicos que patrocinan, y sólo secundariamente en sus reuniones formales. En verdad, en la práctica, el director de un periódico influyente actua por si mismo como un filtro disciplinario, al tamizar los artículos que merecen publicación en su periódico... los periódicos cientificos se cuentan entre las más poderosas "instituciones" de una ciencia. (p. 276)

- Las ciencias del futuro, sin duda, tendrán nuevos voceros, nuevos periódicos y nuevas sociedades; y éstos sólo raramente reemplazarán a sus precedesores por un abrupto coup, o revolución. (p. 277)

- La proliferación de periódicos puede superar las genuinas necesidades disciplinares, asi como el conservadurismo de las organizaciones profesionales puede frenar el apropiado desarrollo disciplinario. (p. 304)

\section{Foro institucional}

Las citas de Toulmin no serán comentadas porque son muy claras y porque fueron presentadas para dar soporte a la idea de que el foro institucional constituído por grupos de referencia, miembros acreditados de la profesión, sociedades científicas y periódicos, es muy importante para la consolidación y evolución de una disciplina. Aunque este foro pueda también frenar el desarrollo disciplinario, su papel en la consolidación y evolución de la disciplina es mucho más importante que el riesgo de frenarla.

La investigación en educación en ciencias parece ya atender los criterios disciplinarios de Toulmin. Esta es la visión optimista de esta presetnación. Sin embargo, hay que reconocer que el foro institucional todavía presenta debilidades. Los grupos de referencia no son muchos, las sociedades también son pocas y los periódicos aunque ya existan en cierta cantidad todavía no tienen criterios muy claros y objectivos. Todo esto está reflectido en las conclusiones y recomendaciones de los Encuentros Iberoamericanos sobre Investigación en Educación Básica realizados en Burgos, en 2002 y 2004, presentados más adelante. 


\section{Cursos de postgrado}

Los criterios disciplinarios de Toulmin no incluyen los cursos de postgrado stricto sensu como componente importante del foro institucional. La creación de dichos cursos es más reciente que la obra de Toulmin. Por ende, no están contemplados. No obstante, en nuestra época dichos cursos, es decir, maestrías (M.Sc.) y Doctorados (Ph.D.), se han convertido en uno de los más relevantes componentes del foro institucional disciplinario. En los días de hoy casi no se puede separar investigación y postgrado. Incluso en las universidades muchas veces hay comisiones, consejos y hasta vicerrectorados de investigación y postgrado.

En ese sentido la "disciplina" investigación en educación en ciencias parece caminar para un fortalecimiento del foro institucional en iberoamérica dado el número creciente de maestrías y doctorados en enseñanza de las ciencias que están surgiendo en los países iberoamericanos, y eso es muy importante.

Pasemos ahora a las conclusiones de los encuentros de investigación antes referidos.

\section{Conclusiones del I y del II EIBIECs}

Durante el I EIBIEC, desarrollado en Burgos, en septiembre de 2002, las siguientes debilidades, desafios y recomendaciones fueron indicados:

\section{Debilidades}

- Pocas lineas de investigación claramente definidas, progresivas. Muchas investigaciones puntuales, muy frecuentemente planificadas a corto plazo, sin grupos fuertes y sin lineas consistentes.

- Proyectos que no definen un problema que sea adecuado para su investigación. Trabajos sin marco teórico, metodológico y epistemológico coherente y consistente. En particular, estudios sin marco teórico o con un supuesto referencial teórico que no se articula con el objeto de estudio ni com los datos y su análisis.

- Fragilidades metodológicas tanto en los enfoques cualitativos como en los cuantitativos. La metodología cualitativa es usada de manera permisiva; parece que bajo la etiqueta "investigación cualitativa" todo vale. Por otro lado, en la metodología cuantitativa a veces se atribuye excesivo peso a ciertas correlaciones o se pone mucha fe en la estadistica, lo que no tiene sentido si no hay un marco teórico por detrás.

- Falta de relevancia de tópicos estudiados. Estudios que no pasan de ser encuestas. Trabajos con concepciones empiristas ingenuas. Investigaciones que parecen no ser propias del área por no tener nada que ver com la ciencia.

- Falta de una visión más compleja de los procesos de aprendizaje, que no son sólo cognitivos. Pocos trabajos sobre evaluación, sin cambiar la concepción de evaluación de los profesores, otros cambios serán aún más difíciles.

- Poco diálogo e interacción con otras comunidades. Es débil el diálogo com los científicos, así como com profesionales de otras áreas que contribuyen en la configuración del marco teórico de la investigación en educación en ciencias, como la psicología y la sociología.

- Referentes teóricos importandos, a veces, de manera acritica, es decir, sin reconstrucción en el ámbito de la educación en ciencias. Existencia de un cierto aplicacionismo. 
- Falta de reconocimiento del área por parte de otras áreas de investigación. Falta de visibilidad frente a los organismos responsables de las politicas educativas, asi como frente al público. Falta de visibilidad en el aula; resultados que no llegan a los docentes.

- Las revistas del área no tienen criterios de calidad bien definidos. La aceptación y rechazo de los artículos son un poco relativos; los pareceres de los consultores muchas veces son muy sintéticos, no son educativos, constructivos.

- Los investigadores del área no aceptan bien la crítica y tampoco la hacen, es decir, falta una tradicción crítica con respecto al área.

- Confusión entre investigación e innovación.

\section{Desafios/Cuestiones}

- ¿Cuál es na laturaleza epistémica de nuestro campo? (¿Una disciplina práctica con dos lealtades: el rigor y la intervención?).

- ¿Cuál es nuestro objeto real de estudio? ¿tiene que ver con los procesos (cognitivos, afectivos, sociales,...) implicados en aquello que llamamos ciencia? ¿Serian las personas implicadas en el área, no necesariamente el contenido cientifico propiamente dicho?

- ¿Cuáles son los problemas relevantes del área? ¿Cuál es su finalidad (¿qué enseñar? ¿qué ciencia enseñar? ¿la de los científicos?'?? ¿El currículum de ciencias? ¿Una teoría del conocimiento profesional docente?

- ¿Cuáles son los critérios de progresividad del área? ¿Cuál es nuestra agenda?

\section{Recomendaciones}

- Mejorar la calidad de las investigaciones - en términos de preguntas relevantes y de fundamentación teórica, metodológica y epistemológica.

- Generar líneas de trabajo progresivas, conducidas por grupos de investigación.

- Incorporar profesores a los grupos de investigación.

- Buscar más visibilidad para el área (en la comunidad académica, en los ministerios, en el aula, en el público,...).

- Mejorar el proceso de evaluación de trabajos por pares. Formar evaluadores. Explicitar criterios de calidad propios del área.

- No confundir investigación e innovación. No confundir investigación y reflexión. No confundir rigor y rigidez formal.

- Tener en cuenta que el conocimiento escolar tiene caracteristicas propias, es decir, no es lo mismo que el conocimiento cientifico; hay que reconstruirlo.

- Aumentar el diálogo con otras áreas (cientificas, sociales y humanas).

- No aislar la investigación en educación en ciencias de la investigación en general.

- Reconstruir las aportaciones externas como, por ejemplo, generar una psicología de las ciencias, una sociología de las ciencias,...

- Estimular la evaluación crítica. Construir criterios para desarrollar esa crítica.

Dichas conclusiones fueron reexaminadas y reiteradas en el II EIBIEC, realizado en Burgos en septiembre de 2004. En estos dos años que se han pasado se pueden observar como puntos positivos: 
- Crecimiento del área, observable por el aumento del número de revistas y de trabajos en congresos. Parte de este crecimiento se debe a la acción de políticas públicas en diferentes paises que han impulsado la creación de nuevos programas de postgrado especificos del área. Podemos asi afirmar que ha habido un aumento cuantitativo importante con la incorporación de nuevos estudiantes y de investigadores de otras áreas.

- Afianzamiento de algunos grupos de investigación, con líneas de investigación más definidas y progresivas.

- Mayor visibilidad del área, con el reconocimiento en algunos paises por parte de las sociedades científicas de sus divisiones de enseñanza como "especialistas" en la enseñanza de las ciencias (reconocimiento de pares).

- Existencia de algunos consensos por lo menos en relación a cómo se aprende.

- Mayor conciencia de la complejidad del área, con un aumento de la riqueza de los referenciales teóricos, metodológicos y epistemológicos utilizados.

Sin embargo,

- Las mismas políticas públicas que en su aspecto positivo han incentivado a los programas y colocado limites temporales para la producción, llevan a una producción apresurada - que no es acorde con las características del área-, privilegiando en algunos casos una visión aplicacionista del conocimiento más que una de producción del conocimiento. Por otra parte, en algunos casos se privilegia el número de miembros de los equipos, que se integran a veces de forma acritica, impidiendo una mayor integración. Esto lleva a una producción de baja calidad y conspira contra la generación de grupos de investigación con líneas de investigación bien definidas y progresivas.

- Faltan aún más grupos de investigación significativos en cada país.

- Muchos de los trabajos continúan aún careciendo de rigor.

- Faltan programas fuertes de formación, con lineas de investigación progresivas.

- Existe una producción desigual en las distintas disciplinas (educación en Física, en Quimica, en Biología,...)

- Se nota una agregación apresurada de investigadores de otras áreas, sin un suficiente conocimiento del área;

- se observa, con cierta frecuencia, una ausencia del "diálogo" con las investigaciones ya realizadas, existiendo una especie de tendencia a "reinventar la rueda".

- Los productos de la investigación aún no tienen un impacto real en las prácticas educativas.

Por esto, además de los desafíos planteados hace dos años y que continúan vigentes,

- Se reafirma la necesidad de la discusión de criterios de calidad. Aunque quizás un criterio primero sea, además de la explicitación del problema, de aspectos teóricos, epistemológicos y metodológicos, la explicitación de cómo éstos se relacionan con los resultados obtenidos, es necesaria la discusión con las distintas asociaciones del área de estos criterios de calidad y el levantamiento de consensos minimos en relación a esto. En este sentido, proponemos que este documento sirva de base para una discusión que nos pueda permitir en dos años más construir algunos de estos criterios. Por otra parte, consideramos necesaria la explicitación de los criterios de evaluación usados por los organismos oficiales en relación al área, congresos y revistas, de forma que aporte más elementos para esta discusión. 
- Es necesaria una reafirmación del área en términos de financiamiento, con la defensa de un espacio propio para los financiamientos de Ciencia e Tecnología;

- Es necesario un crecimiento de la innovación - o sea, la necesidad de traspaso de los productos de la investigación a las prácticas educativas, convirtiendose en uno de los mayores desafios del área;

- Es necesária la existencia del área con "nombre propio" en los organismos de financiamento oficiales, que posibiliten una competencia en el mismo nivel con otras áreas.

\section{Conclusión}

En esta presentación mi postura hacia la investigación en educación en ciencias ha sido bastante crítica. Sin embargo, no es pesimista. Al revés, es optimista y constructiva. Creo que dicha investigación - que ya tiene sus treinta años, por lo menos - há crecido mucho, se está consolidando y puede ya atender a criterios disciplinarios como los propuestos por Stephen Toulmin (1977). Por otro lado, dicho crecimiento no debe ser desordenado, al azar, y dicha consolidación depende mucho de crítica y de lo que Toulmin llama de foro institucional.

Nuestros grupos de referencia, los miembros acreditados de nuestra comunidad, las asociaciones, periódicos y congresos de investigación, así como las maestrías y doctorados en enseñanza de las ciencias, tienen un papel fundamental en el crecimiento y consolidación de esta nueva disciplina que hemos creado.

\section{Referências}

MOREIRA, M. A. A pesquisa em educação em ciências e a formação permanente do professor de ciências. In: CONGRESO IBEROAMERICANO DE EDUCACIÓN EN CIENCIAS EXPERIMENTALES, 1, 1998, La Serena. Actas... La Serena: [s.n.], 1998.

MOREIRA, M. A. Investigación básica en educación en ciencias: una visión personal. Revista Chilena de Educación Científica, Santiago, v. 3, n. 1, p. 10-17, 2004.

TOULMIN, S. E. La comprensión humana: el uso colectivo y la evolución de los conceptos. Madrid, Alianza, $1977.523 \mathrm{p}$.

Artigo recebido em dezembro de 2004 e selecionado para publicação em julho de 2005. 